\title{
What brings pericytes to tumor vessels?
}

\author{
Rakesh K. Jain and Michael F. Booth
}

Edwin L. Steele Laboratory, Department of Radiation Oncology, Massachusetts General Hospital and Harvard Medical School, Boston, Massachusetts, USA

Paracrine signaling via platelet-derived growth factor B (PDGFB), expressed by endothelial cells, and its receptor PDGFR- $\beta$, expressed by pericytes, plays a central role in blood vessel maturation. A new study (see the related article beginning on page 1142) reveals that it is not just the presence of PDGFB, but how it is presented to pericytes, that determines the quality of the endothelium-pericyte interaction.

J. Clin. Invest. 112:1134-1136 (2003). doi:10.1172/JCI200320087.

Tumor vessels are enigmatic. Their wall structure and branching patterns are abnormal (1). The mural cells (MCs) pericytes and VSMCs - are either absent or loosely associated with the tumor endothelium. This abnormality contributes to the leakiness of the vessels, which, counterintuitively, poses a challenge for drug delivery (2). At the same time, this very absence of MCs renders the vessels vulnerable to antiangiogenic therapies. Thus, an understanding of the molecular mechanism of MC recruitment to tumor endothelial cells (ECs) is important for cancer treatment.

The tumor vascular network is formed by vasculogenesis (de novo vessel formation from angioblasts or stem cells) as well as angiogenesis (sprouting, bridging, and intussusceptive growth from existing vessels). VEGF signaling initiates the formation of new vessels by recruiting ECs to form tubes. VEGF also triggers a chain of molecular and cellular events that stabilize the EC tubes by recruiting MCs and generating an ECM. At least four

\footnotetext{
Address correspondence to: Rakesh K. Jain, Edwin L. Steele Laboratory, Department of Radiation Oncology, 100 Blossom Street, Boston, Massachusetts 02114, USA. Phone: (617) 726-4083, Fax: (617) 724-1819; E-mail: jain@steele.mgh.harvard.edu. Conflict of interest: The authors have declared that no conflict of interest exists. Nonstandard abbreviations used: mural cell (MC); endothelial cell (EC); sphingosine-1phosphate-1 (S1P1); endothelial differentiation sphingolipid Gprotein-coupled receptor 1 (EDG1).
}

molecular pathways are involved in the regulation of this process, and the key components of these include (a) platelet-derived growth factor $B$ (PDGFB) and PDGF receptor $\beta$ (PDGFR- $\beta$ ); (b) sphingosine-1-phosphate-1 (S1P1) and endothelial differentiation sphingolipid G-proteincoupled receptor 1 (EDG1); (c) Ang1 and Tie 2 ; and (d) TGF- $\beta$ (1). Based on the phenotype of Pdgfb-and Pdgfr- $\beta$ null mice, Hellstrom et al. previously demonstrated that paracrine signaling via PDGFB (expressed by ECs) and its receptor PDGFR- $\beta$ (expressed by MCs) plays a central role in $\mathrm{MC}$ recruitment and blood vessel stabilization (3). However, the precise mechanism underlying the recruitment of MCs to ECs is shrouded in mystery.

In this issue of the JCI, Abramsson et al. (4) propose that it is not just the presence of PDGFB but how it is presented to MCs that determines the quality of EC-MC interactions. They offer compelling evidence for the hypothesis that an extracellular gradient of PDGFB, adjacent to the ECs, coaxes MCs into close contact with vessels (4). Because technical difficulties precluded the direct measurement of extracellular PDGFB concentration gradients in vivo, Abramsson et al. (4) disrupted these gradients by a series of clever approaches (Figure 1) and monitored the effect on MC recruitment.

The PDGFB molecule contains a "retention motif," a region that mediates binding to proteoglycans at the cell surface and in the ECM.
Presumably, this motif helps to localize PDGFB in or near the secreting ECs (Figure 1, a and b). Abramsson et al. (4) demonstrate that when T241 fibrosarcoma cells, which do not express PDGFB, are implanted in wild-type mice, the resulting tumor vessels are invested with MCs. When the same tumor cells are transplanted into $p d g f b^{r e t}$ /ret mice - transgenic mice that express a form of PDGFB that lacks the retention motif (5) - they have fewer vessel-associated MCs and looser attachment between MCs and ECs (4). This is presumably because the modified PDGFB is free to diffuse throughout the ECM and does not form sharp, EC-associated gradients (Figure 1c). If tumor cells expressing PDGFB are used, the number of recruited MCs increases, but since the extra PDGFB is not concentrated around the ECs, the EC-MC attachment in $p d g f f^{\text {ret }}$ ret mice does not improve (Figure 1d). Finally, exogenous cells that do not express PDGFR- $\beta$ are unable to form contact with ECs (Figure 1e).

\section{Pericyte coverage: bad or good?}

These findings have important clinical implications, not only for cancer, but also for other diseases characterized by abnormal EC-MC interactions (6). The host cells (possibly MCs) that surround the tumor vessels are known to produce VEGF (7, 8) (Figure 1f), which is a survival factor for ECs. Thus it seems reasonable to speculate that MCs serve as a private source of VEGF for the adjacent ECs (9). If the MCs were absent or could not produce VEGF, the endothelium would become vulnerable to VEGF blockade. Indeed, this is the case for both normal and pathological vessels (10). Furthermore, tyrosine kinase inhibitors, which block multiple kinases including PDGFR- $\beta$, enhance the effect of VEGF inhibitors (11). This enhancement may be a result of disrupted EC-MC contact, or it may be caused by the reduced production of VEGF and/or tumor matrix molecules by PDGFR- $\beta$-positive perivascular cells. 

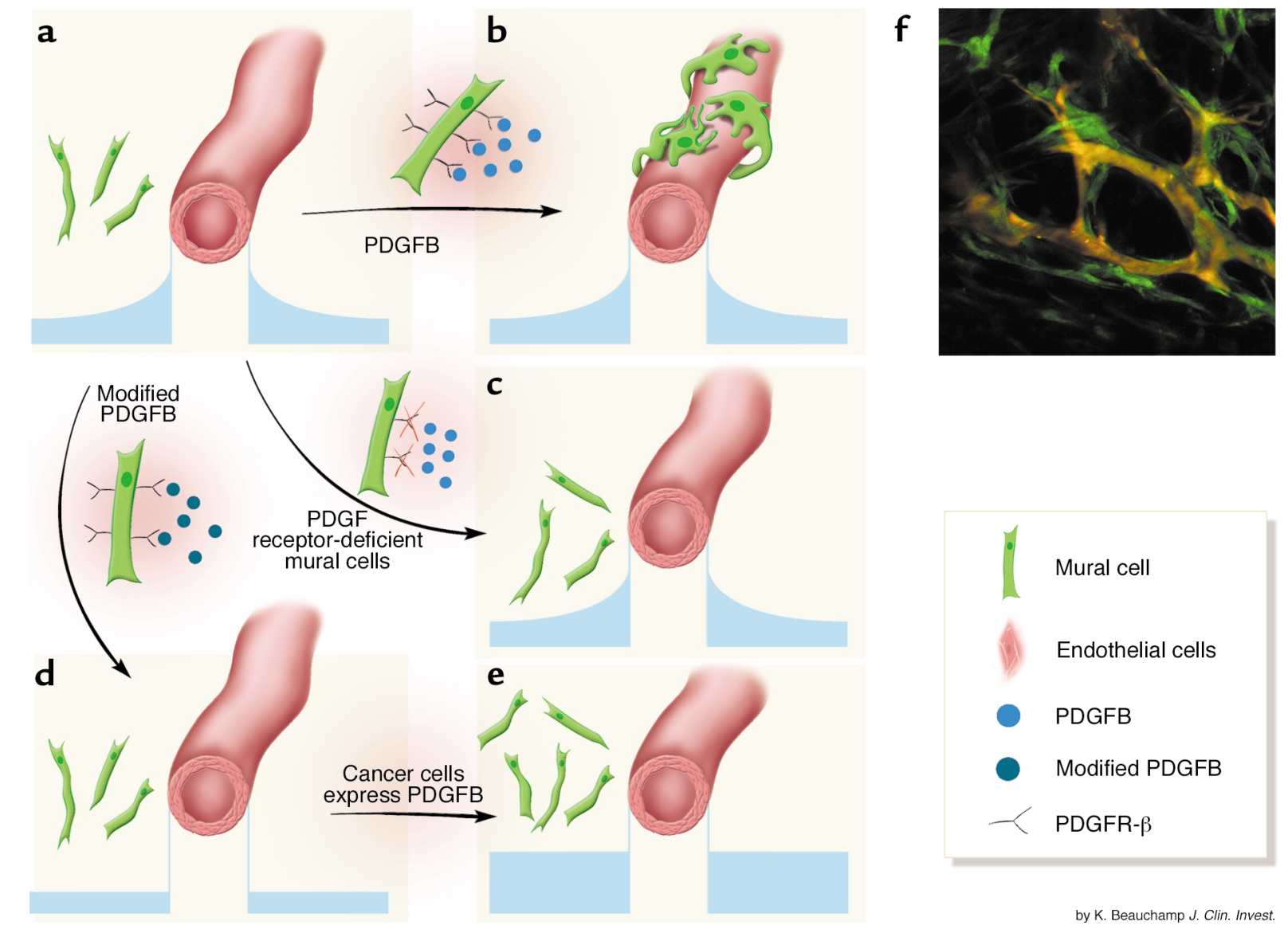

Figure 1

MC recruitment to tumor vessels depends on a putative gradient of PDGFB. (a) PDGFB, secreted by an EC tube, has higher concentration near the tube, as shown in the small graphs. (b) Normally, MCs, which express PDGFR- $\beta$, sense the PDGFB concentration gradient and move in to closely contact the ECs. (The basement membrane is not shown.) (c) If MCs do not express PDGFR- $\beta$, then they are not recruited. (d) Modifying PDGFB to remove its matrix retention eliminates the PDGFB concentration gradient and results in poor EC-MC contact. (e) If tumor cells express PDGFB, the PDGFB concentration in the tumor overwhelms the peri-EC gradient and EC-MC contact remains poor. ( $f$ ) Perivascular cells expressing GFP under the control of the VEGF promoter surround vessels in the tumor interior. The image was obtained using a two-photon microscope. Orange, vessel lumens; green, MCs. Panel f is reproduced with permission from Nature Medicine (8).

When antiangiogenic therapy prunes away tumor vessels with little or no MC coverage, it may leave behind a "normalized" network of MC-invested vessels. This normalization process could be exploited for the improved delivery of drugs to tumors (12). Because recent clinical trials suggest that, for the near future, antiangiogenic therapies must be combined with cytotoxic therapies to achieve the best therapeutic response (13), the normalization of tumor vasculature - and the role of MCs in that normalization - is a fertile area of research.

Although recent efforts have focused on destabilizing tumor vessels by interfering with EC-MC communication, the other side of the coin - enhancing EC-MC communi- cation - is equally important in medicine. The formation of a mature, well-organized, stable vasculature is a key goal in tissue engineering, regenerative medicine, therapeutic angiogenesis, and the treatment of vascular diseases such as diabetic retinopathy $(1,6)$.

\section{Unanswered questions}

As any good study does, the present work (4) raises many questions. For example, where do the MCs in tumors come from? Does TGF- $\beta$ (or another trigger) prompt fibroblasts at the tumor/host interface to differentiate into myofibroblasts and then into pericyte-like cells $(1,14,15)$ ? How do the PDGF gradients produce a close association between ECs and
MCs? Do they direct subcellular localization of adhesion molecules, localized ECM production, or other factors, as Abramsson and colleagues suggest (4)? Vessels in most tumors either lack MC coverage or have abnormal MC coverage. Is this due to the presence of nonretained isoforms of PDGFB? Or is it that PDGFB levels in tumors are so large that they overwhelm the peri-EC gradients? Finally, how does the PDGFB/ PDGFR- $\beta$ pathway interact with the other molecular pathways that are known to play a role in EC-MC interactions? These include VEGF, TGF- $\beta$, ephrins, Ang1 and Tie2, and S1P1 and EDG1 (1).

An even more fundamental question is: What is the structural and 
functional significance of $\mathrm{MC}$ coverage and EC-MC association in tumors? Mere physical proximity does not guarantee proper molecular interaction or microstructural integration between MCs and ECs. For example, despite their tight EC-MC association (4), T241 fibrosarcomas have high vascular permeability (16). Furthermore, improved MC coverage does not guarantee normal vascular function. For example, $80 \%$ of the vascular surface area of the murine mammary carcinoma MCaIV is covered with MCs (17), yet these tumor vessels are profoundly leaky (18). Is it possible that the tumor vessel-associated MCs increase the vessel permeability by expressing VEGF? In addition to controlling vessel function and integrity, do these perivascular cells lead endothelial sprouts during angiogenesis, as suggested by both intravital microscopy and immunohistochemistry $(8,17)$ ? The present study is an important step in answering these urgent questions.
1.Jain, R.K. 2003. Molecular regulation of vessel maturation. Nat. Med. 9:685-693.

2. Jain, R.K. 1994. Barriers to drug delivery in solid tumors. Sci. Am. 271:58-65.

3. Hellstrom, M., et al. 2001. Lack of pericytes leads to endothelial hyperplasia and abnormal vascular morphogenesis. J. Cell Biol. 153:543-553.

4. Abramsson, A., Lindblom, P., and Betsholtz, C. 2003. Endothelial and nonendothelial sources of PDGF-B regulate pericyte recruitment and influence vascular pattern formation in tumors. J. Clin. Invest. 112:1142-1151. doi:10.1172/JCI200318549.

5. Lindblom, P., et al. 2003. Endothelial PDGF-B retention is required for proper investment of pericytes in the microvessel wall. Genes Dev. 17:1835-1840.

6. Carmeliet, P. 2003. Angiogenesis in health and disease. Nat. Med. 9:653-660.

7. Fukumura, D., et al. 1998. Tumor induction of VEGF promoter activity in stromal cells. Cell. 94:715-725

8. Brown, E.B., et al. 2001. In vivo measurement of gene expression, angiogenesis and physiological function in tumors using multiphoton laser scanning microscopy. Nat. Med. 7:864-868.

9. Reinmuth, N., et al. 2001. Induction of VEGF in perivascular cells defines a potential paracrine mechanism for endothelial cell survival. FASEBJ. 15:1239-1241.

10. Benjamin, L.E., Golijanin, D., Itin, A., Pode, D. and Keshet, E. 1999. Selective ablation of immature blood vessels in established human tumors follows vascular endothelial growth factor withdrawal. J. Clin. Invest. 103:159-165.

11. Bergers, G., Song, S., Meyer-Morse, N., Bergsland, E., and Hanahan, D. 2003. Benefits of targeting both pericytes and endothelial cells in the tumor vasculature with kinase inhibitors. J. Clin. Invest. 111:1287-1295. doi:10.1172/JCI200317929.

12. Jain, R.K. 2001. Normalizing tumor vasculature with anti-angiogenic therapy: a new paradigm for combination therapy. Nat. Med. 7:987-989.

13. Hurwitz, H., et al. 2003. Bevacizumab (Avastin, a monoclonal antibody to vascular endothelial growth factor) prolongs survival in first-line colorectal cancer (CRC): results of a phase III trial of bevacizumab in combination with bolus IFL (irinotecan, 5-fluorouracil, leucovorin) as first-line therapy in subjects with metastatic CRC. Proceedings of the American Society of Clinical Oncology. 22:2107. (Abstr.)

14. Hirschi, K.K., Rohovsky, S.A., and D'Amore, P.A. 1998. PDGF, TGF-beta, and heterotypic cell-cell interactions mediate endothelial cell-induced recruitment of 10T1/2 cells and their differentiation to a smooth muscle fate. J. Cell Biol. 141:805-814.

15. Chambers, R.C., Leoni, P., Kaminski, N., Laurent, G.J., and Heller, R.A. 2003. Global expression profiling of fibroblast responses to transforming growth factor-beta1 reveals the induction of inhibitor of differentiation-1 and provides evidence of smooth muscle cell phenotypic switching. Am. J. Pathol. 162:533-546.

16. Kadambi, A., et al. 2001. Vascular endothelial growth factor (VEGF)-C differentially affects tumor vascular function and leukocyte recruitment: role of VEGF-receptor 2 and host VEGF-A Cancer Res. 61:2404-2408.

17. Morikawa, S., et al. 2002. Abnormalities in pericytes on blood vessels and endothelial sprouts in tumors. Am. J. Pathol. 160:985-1000.

18. Hobbs, S.K., et al. 1998. Regulation of transport pathways in tumor vessels: role of tumor type and microenvironment. Proc. Natl. Acad. Sci. U. S. A. 95:4607-4612

\section{Receptor "cross talk" in innate immunity}

\section{Eicke Latz and Douglas T. Golenbock}

Division of Infectious Diseases and Immunology, University of Massachusetts Medical School, Worcester, Massachusetts, USA

Toll-like receptors (TLRs) recognize microbial molecular signatures and can initiate innate immune responses against invading pathogens. A new study (see the related article beginning on page 1234) reports how TLR2 expression by endothelia is locally upregulated by the action of activated polymorphonuclear neutrophils via an unprecedented mechanism involving cell-cell interaction and $\mathrm{NAD}(\mathrm{P}) \mathrm{H}$ oxidase. The report reveals yet another way in which the primordial innate immune system is remarkably complex.

J. Clin. Invest. 112:1136-1137 (2003). doi:10.1172/JCI200320040.
In this issue of the JCI, Fan et al. report on receptor "cross talk" between members of the Toll-like receptor (TLR) family (1). This elegant study confirms previous observations that inflammation via TLR4 results in the enhanced expression of TLR2 $(2,3)$. However, Fan et al. elucidate a new mechanism of enhancement of endothelial TLR2 expression that may have important physiological consequences. Polymor- phonuclear neutrophils (PMNs) that have been activated by endotoxin (LPS) can instruct endothelia to upregulate TLR2 and thus sensitize endothelia to TLR2 ligands. This message is sent to endothelial cells by the release of free oxygen radicals as the result of a CD18dependent cell-cell interaction. TLR2 expression in endothelium, for example, was dramatically enhanced when endothelium was co-incubated with activated PMN from normal mice but not mice with a targeted lesion in $g p^{91 p h o x}$, a member of the NAD(P)H oxidase complex. The enhanced TLR2 expression was demonstrated to result in subsequently enhanced responses to peptidoglycan, a TLR2 ligand. The ability of LPS to sensitize endothelial cells for TLR2 stimuli via LPS-induced activation of PMNs represents a previously unsuspected positive feedback loop. What distinguishes this paper by Fan et al. from a large number of reports that purport to demonstrate receptor cross talk" in the innate immune system is that the authors go on to demonstrate that the relationship among TLR4, TLR2, and the oxidative machinery has functional consequences. Dur- 